\title{
Declínio Cognitivo de Etiologia Medicamentosa
}

\author{
Cognitive Decline of Medicamentous Etiology
}

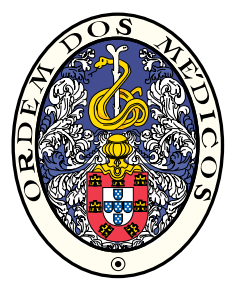

Luís CALDEIRA ${ }^{1}$, Sara VARANDA², Álvaro MACHADO², Carla FERREIRA², Gisela CARNEIRO

Acta Med Port 2014 Mar-Apr;27(2):266-268

\section{RESUMO}

Nos doentes idosos há um risco elevado de medicação inapropriada e de efeitos adversos da polimedicação. Doente do sexo feminino, 68 anos, previamente autónoma, recorre ao Serviço de Urgência por suspeita de Acidente Vascular Cerebral. Segundo o marido, nos seis meses prévios à admissão, apresentou progressiva dependência funcional e períodos de desorientação. Por esse motivo, havia recorrido a consultas de diferentes especialidades, encontrando-se polimedicada, sendo impossível perceber a posologia administrada. Apresentava-se desatenta, desorientada, apráxica, com mioclonias e marcha atáxica. Durante o internamento, após suspensão de toda a medicação crónica, revelou melhoria gradual, apresentando, à data de alta, exame neurológico completamente normal. A iatrogenia medicamentosa como causa reversível para quadros demenciais deve ser equacionada. Todos os doentes, particularmente os idosos e seus cuidadores, deverão ser adequadamente informados acerca dos medicamentos prescritos e da respetiva posologia. A utilização do modelo biopsicossocial poderá evitar a polimedicação inapropriada e a iatrogenia.

Palavras-chave: Demência/induzida quimicamente; Doença latrogénica; Perturbações Cognitivas; Polimedicação; Portugal.

\section{ABSTRACT}

In the elderly there is a high risk of inappropriate medication and adverse effects of polypharmacy. A 68 year-old female patient resorted to the Emergency Room for suspected stroke. According to the husband, in the six months prior to admission, she became progressively disorientated and dependent. She had resorted to various appointments from different specialties and was polymedicated. It was impossible to clarify the exact dosage. On neurological examination she presented disturbance in attention and memory, disorientation, constructional apraxia, myoclonus and gait imbalance. After the suspension of all chronic medication, she showed gradual improvement. By the time of discharge, her neurological examination was completely normal. latrogenic effect of drugs as a cause of reversible dementia should be considered. All patients, particularly the elderly and their caregivers, should be suitably informed about the drugs that are prescribed and the dosages used. Using the biopsychosocial model could prevent inappropriate polypharmacy and iatrogeny. Keywords: Cognition Disorders; Dementia/chemically induced; latrogenic Disease; Polypharmacy.

\section{INTRODUÇÃo}

Em Portugal, a população residente com 65 anos ou mais ultrapassou, em 2011, os dois milhões. ${ }^{1}$ A este fenómeno associa-se um aumento da prevalência e incidência de patologias características desta faixa etária, com necessidade de consultas regulares e polimedicação. Para superar o desafio do envelhecimento deve garantir-se que as pessoas se mantenham saudáveis, ativas e autónomas durante o máximo tempo possível. ${ }^{2}$

A demência caracteriza-se por défice cognitivo que inclui compromisso da memória e pelo menos um dos seguintes: afasia, apraxia, agnosia e/ou perturbação da capacidade executiva. Deve ser suficientemente severo para comprometer o funcionamento ocupacional ou social e representar um declínio relativamente a um nível prévio de funcionamento. Não pode ocorrer exclusivamente durante o curso de um delirium, caracterizado por perturbação da consciência desenvolvida num curto período de tempo (horas a dias), flutuante ao longo do dia e sendo consequência fisiológica direta de um estado físico geral. ${ }^{3} \mathrm{~A}$ definição de demência do "Diagnostic and Statistical Manual of Mental Disorders" baseia-se num padrão de défices cognitivos e não envolve qualquer conotação prognóstica, podendo ser progressiva, estática ou remitente. A forma de início, o curso subsequente e, particularmente, a reversibilidade ocorrem em função da patogenia e da disponibilidade e aplicação oportuna de um tratamento eficaz. Confirmando esta noção, um estudo prospetivo apontou para uma prevalência de $19 \%$ de etiologias potencialmente reversíveis de demência. ${ }^{4}$

\section{CASO CLÍNICO}

Doente do sexo feminino, 68 anos, dextra e previamente autónoma, é trazida ao Serviço de Urgência (SU) hospitalar com ativação da Via Verde Acidente Vascular Cerebral (AVC) por, subitamente, ter sido notada pelo filho incapacidade da doente para utilizar os talheres com a mão direita.

No SU, o marido, única pessoa com quem convivia diariamente, descrevia o início dos sintomas seis meses antes. A doente mostrava dificuldades em realizar tarefas habituais - queimava a comida, precisava de ajuda no banho, confundia os dias e nem sempre sabia onde estava. Com o agravamento progressivo da sintomatologia, foi observada pelo médico de família, bem como em consultas particulares de outras especialidades, nas quais se insti-

1. Medicina Geral e Familiar. Unidade de Saúde Familiar Aquae Flaviae. Chaves. Portugal.

2. Serviço de Neurologia. Hospital de Braga. Braga. Portugal.

Recebido: 23 de Fevereiro de 2013 - Aceite: 09 de Setembro de 2013 | Copyright @ Ordem dos Médicos 2014 
tuíram sucessivas alterações terapêuticas. O marido desconhecia a medicação da doente, dado que a sua gestão continuava sob responsabilidade desta. Apresentou vários medicamentos: sinvastatina (duas embalagens de marcas diferentes), paroxetina (três embalagens de marcas diferentes), alprazolam, brometo de clidínio clorodiazepóxido, beta-histina, deflazacorte, omeprazol, trazodona, propranolol, citicolina, trimetazidina, alfuzosina, paracetamol, brometo de otilónio, aceclofenac, celecoxib, etoricoxib, glucosamina e tri-hexifenidilo.

Ao exame neurológico encontrava-se acordada, desatenta e bradipsíquica. Nomeava, repetia e cumpria ordens simples. Estava desorientada no tempo, espaço e alopsiquicamente, dizendo que estava 'à espera da vez para cortar o cabelo' (sic). Dirigia o olhar, sem limitações da oculomotricidade. Elevava os quatro membros simetricamente, todos apresentando mioclonias multifocais negativas. Os reflexos osteotendinosos eram de limiar baixo, simétricos e com reflexo cutâneo-plantar flexor, bilateralmente. A marcha autónoma era impossível por desequilíbrio. Não apresentava sinais meníngeos.

Realizou tomografia computorizada crânio-encefálica e estudo analítico sumário que não apresentavam alterações significativas.

Por conseguinte, foi desativada a Via Verde AVC, colocando-se como hipótese diagnóstica declínio cognitivo rapidamente progressivo, de causa indeterminada. Foi internada no Serviço de Neurologia para investigação etiológica.

Nos primeiros três dias, a doente manteve estado neurológico sobreponível. Procedeu-se a uma avaliação neurocognitiva mais detalhada, constatando-se persistência da desorientação, comportamento de utilização, perseveração, apraxia construcional e remissão das mioclonias. Pontuava 16 em 30 no Mini-Mental State Examination (MMSE). Após melhoria gradual, ao sexto dia de internamento, a doente conseguiu especificar como geria o seu regime terapêutico: paroxetina $90 \mathrm{mg} / \mathrm{dia}$, sinvastatina $40 \mathrm{mg} / \mathrm{dia}$, alprazolam $1 \mathrm{mg} /$ dia, brometo de clidínio $2.5 \mathrm{mg} / \mathrm{dia}$, clorodiazepóxido $5 \mathrm{mg} / \mathrm{dia}$, beta-histina $16 \mathrm{mg} / \mathrm{dia}$, deflazacorte $3 \mathrm{mg} / \mathrm{dia}$, omeprazol $20 \mathrm{mg} / \mathrm{dia}$, trazodona $150 \mathrm{mg} / \mathrm{dia}$, propranolol $40 \mathrm{mg} / \mathrm{dia}$, citicolina $600 \mathrm{mg} / \mathrm{dia}$, trimetazidina $20 \mathrm{mg} / \mathrm{dia}$, alfuzosina $5 \mathrm{mg} / \mathrm{dia}$, brometo de otilónio $40 \mathrm{mg} / \mathrm{dia}$, aceclofenac $100 \mathrm{mg} / \mathrm{dia}$, celecoxib $200 \mathrm{mg} / \mathrm{dia}$, etoricoxib $90 \mathrm{mg} /$ dia, glucosamina $1500 \mathrm{mg} / \mathrm{dia}$ e tri-hexifenidilo $4 \mathrm{mg} / \mathrm{dia}$.

Realizou-se estudo analítico alargado, incluindo doseamentos de vitamina B12, ácido fólico, perfil tiroideu, hormona paratiroideia, teste Venereal Disease Research Laboratory e serologia anti-Vírus da Imunodeficiência Humana, com resultados normais ou negativos. A ressonância magnética crânio-encefálica não evidenciou alterações. $O$ eletroencefalograma, realizado após 72 horas de internamento, demonstrou actividade de base alfa, sem alterações patológicas. A única atitude terapêutica consistiu na suspensão de toda a medicação crónica da doente. Ao nono dia teve alta, com funções superiores preservadas, pon- tuando 29 no MMSE. A proposta terapêutica ambulatória consistiu em manter omeprazol e sinvastatina, suspendendo os restantes medicamentos. Foi orientada para consulta de Neurologia, para excluir défice cognitivo de base ao qual se tenha sobreposto a etiologia tóxica.

\section{DISCUSSÃO}

A iatrogenia medicamentosa deve equacionar-se como causa reversível de um quadro demencial, sobretudo numa população envelhecida e polimedicada. Os fármacos que, per si ou mediante interação, poderão originar deterioração cognitiva são numerosos.

Os anticolinérgicos podem ter efeitos adversos, especialmente na população idosa. ${ }^{5}$ Este grupo é particularmente suscetível devido à redução fisiológica da celularidade dos núcleos colinérgicos, da densidade dos recetores muscarínicos e ao aumento da permeabilidade da barreira hemato-encefálica (BHE). A toxicidade pode ser facilitada pela redução do metabolismo hepático e excreção renal. ${ }^{6,7}$ Os seus efeitos adversos neurológicos agudos consistem em manifestações cognitivas (confusão, agitação, ansiedade), extrapiramidais (tremor) e hípnicas (sonolência, insónia, pesadelos). O seu equivalente crónico traduz-se em défice cognitivo e demência. ${ }^{8}$ Os opióides, antidepressivos tricíclicos, inibidores seletivos da recaptação da serotonina, antipsicóticos, benzodiazepinas, anti-histamínicos, anti-eméticos, anti-espasmódicos, corticosteróides, broncodilatadores, anti-hipertensores, diuréticos, digitálicos, anti-arrítmicos e anticoagulantes orais podem também exercer ação anticolinérgica. ${ }^{6,8,9}$ As estatinas poderão associar-se a deterioração cognitiva, com risco superior para as lipofílicas, que atravessam mais facilmente a BHE. ${ }^{10}$

Neste caso é complexo estabelecer relações causais inequívocas entre fármacos específicos e sinais neurológicos, atendendo ao elevado número de medicamentos, alguns em dose supra-terapêutica. Terão sido, presumivelmente, os antidepressivos, bem como a sua interacção, a justificar o surgimento de mioclonias negativas, através de um aumento da transmissão serotoninérgica. O mesmo mecanismo poderá explicar o achado de hiperreflexia osteotendinosa. ${ }^{11} \mathrm{~A}$ ataxia atribui-se provavelmente à toma de duas benzodiazepinas. Estes fármacos, em associação à sinvastatina e ao tri-hexifenidilo, terão tido impacto na desatenção, desorientação e lentidão psíquica evidenciadas.

A deterioração cognitiva poderia ter sido evitada se houvesse uma melhor articulação entre os Cuidados de Saúde Primários e Secundários e tivesse sido utilizado o modelo biopsicossocial nas consultas.

\section{CONFLITOS DE INTERESSE}

Os autores declaram a inexistência de conflitos de interesse na realização do presente trabalho.

\section{FONTES DE FINANCIAMENTO}

Não existiram fontes externas de financiamento para a realização deste artigo. 
REFERENCIAS

1. Instituto Nacional de Estatística. Censos 2011 [Consultado 2012 Nov 28] Disponível em: http://censos.ine.pt/xportal/xmain?xpid=CENSOS\&x pgid=censos2011_apresentacao.

2. Ministério da Saúde, Direcção Geral da Saúde. Programa Nacional para a Saúde das Pessoas Idosas. Lisboa: DGS; 2004. [Consultado 2012 Nov 28] Disponível em: http://www.min-saude.pt/NR/rdonlyres/1C6DFF0E9E74-4DED-94A9-F7EA0B3760AA/0/i006346.pdf.

3. Delirium, demência, perturbações mnésicas e outras perturbações cognitivas. In Manual de diagnóstico e estatística das perturbações mentais. $4^{\mathrm{a}}$ ed. Lisboa: Climepsi editores; 2006.

4. Hejl A, Høgh P, Waldemar G. Potencially reversible conditions in 1000 consecutive memory clinic patients. J Neurol Neurosurg Psychiatr. 2002;73:390-4.

5. Rigo JC, Rigo JF, Faria BC, Santos VM. Demência reversível e quedas associadas ao biperideno. Rev Psiq Clín. 2006;33:24-7.

6. Robles Bayón A, Gude Sampedro F. Prescripciones inconvenientes en el tratamiento del paciente con deterioro cognitivo. Neurología. 2012 (in press).
7. Campbell N, Boustani M, Limbil T, Ott C, Fox C, Maidment I, et al. The cognitive impact of anticholinergics: A clinical review. Clin Interv Aging. 2009;4:225-33.

8. Ancelin ML, Artero S, Portet F, Dupuy AM, Touchon J, Ritchie K. Nondegenerative mild cognitive impairment in elderly people and use of anticholinergic drugs: longitudinal cohort study. BMJ. 2006;332:455-9.

9. Carrièrre I, Fourrier-Reglat A, Dartigues JF, Rouaud O, Pasquier F, Ritchie K, et al. Drugs with anticholinergic properties, cognitive decline, and dementia in an elderly general population: the 3-city study. Arch Intern Med. 2009;169:1317-24.

10. Rojas-Hernandez $\mathrm{CH}$, Cameron JCF. Is statin-associated cognitive impairment clinically relevant? A narrative review and clinical recommendations. Ann Pharmacother. 2012;46:549-57.

11. Jiménez-Jiménez FJ, Puertas I, de Toledo-Heras M. Drug-induced myoclonus: frequency, mechanisms and management. CNS Drugs. 2004;18:93-104.

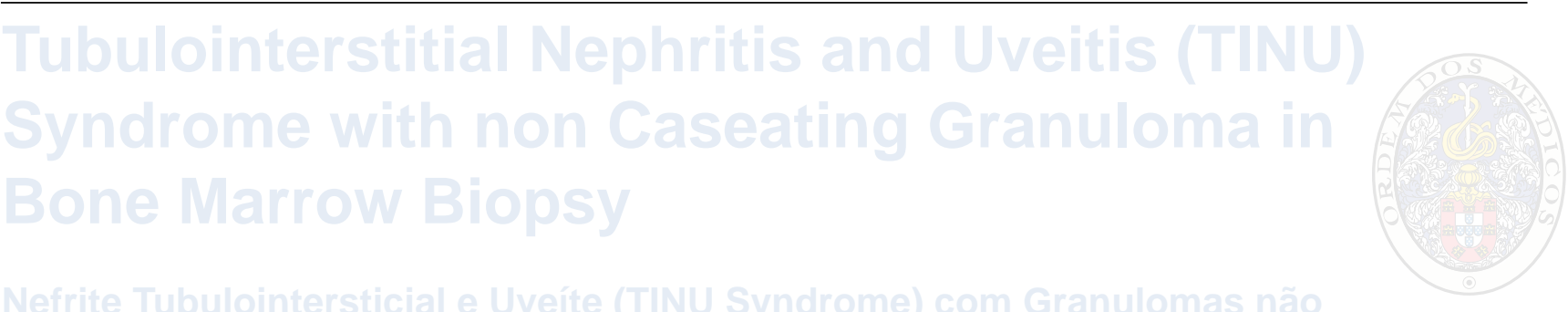

\section{Caseosos na Biópsia Óssea}




\section{Declínio Cognitivo de Etiologia Medicamentosa} Acta Med Port 2014:27:266-268

Publicado pela Acta Médica Portuguesa, a Revista Científica da Ordem dos Médicos

Av. Almirante Gago Coutinho, 151

1749-084 Lisboa, Portugal.

Tel: +351218428215

E-mail: submissao@actamedicaportuguesa.com

www.actamedicaportuguesa.com

ISSN:0870-399X | e-ISSN: 1646-0758

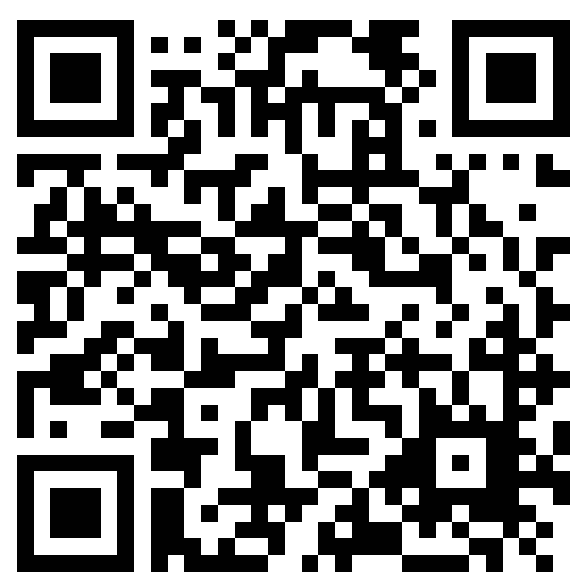

